\title{
Improving the accuracy of predicting protein-ligand binding-free energy with semiempirical quantum chemistry charge
}

\author{
Cheng Peng ${ }^{1,2}$, Jinan Wang*,1, Yuqi Yu' ${ }^{1,2}$, Guimin Wang ${ }^{1,2}$, Zhaoqiang Chen ${ }^{1,2}$, Zhijian \\ $\mathrm{Xu}^{1}$, Tingting Cai ${ }^{1}$, Qiang Shao ${ }^{1}$, Jiye $\mathrm{Shi}^{3}$ \& Weiliang Zhu**,1,2,4 \\ ${ }^{1}$ Drug Discovery \& Design Center, CAS Key Laboratory of Receptor Research, Shanghai Institute of Materia Medica, Chinese \\ Academy of Sciences, 555 Zuchongzhi Road, Shanghai, 201203, PR China \\ ${ }^{2}$ School of Pharmacy, University of Chinese Academy of Sciences, No.19A Yuquan Road, Beijing, 100049, PR China \\ ${ }^{3}$ UCB Biopharma SPRL, Chemin du Foriest, Braine-l'Alleud, Belgium \\ ${ }^{4}$ Open Studio for Druggability Research of Marine Natural Products, Pilot National Laboratory for Marine Science and Technology \\ (Qingdao), 1 Wenhai Road, Aoshanwei, Jimo, Qingdao, 266237, PR China. \\ *Author for correspondence: jawang@simm.ac.cn \\ **Author for correspondence: wlzhu@simm.ac.cn
}

Aim: It is a challenge to predict binding-free energy $(\Delta G)$ accurately. Methodology/results: For accurate $\Delta G$ prediction, a new strategy combining solvated interaction energy (SIE) or molecular mechanics/generalized Born surface area (MM/GBSA) approach with the Coulson charge of both protein and ligand calculated by semiempirical quantum mechanics (SQM), named SIE-SQMPC or MM/GBSASQMPC approach, was developed and tested on 50 protein-ligand complexes. Both approaches achieved higher correlation $\left(R^{2}\right)$ between experimental and predicted $\Delta G$ than that with Amber-ff03 charge, even for ligands with highly different scaffolds. But, SIE-SQMPC is computationally much faster than MM/GBSASQMPC. Conclusion: SIE-SQMPC provided an effective alternative to predict $\Delta G$ of protein-ligand binding $\left(R^{2}=0.66-0.94\right.$ for SIE-AM1; $R^{2}=0.59-0.98$ for SIE-PM7), which has the potential of high-throughput processing for molecular docking and drug design.

\section{Graphical abstract:}

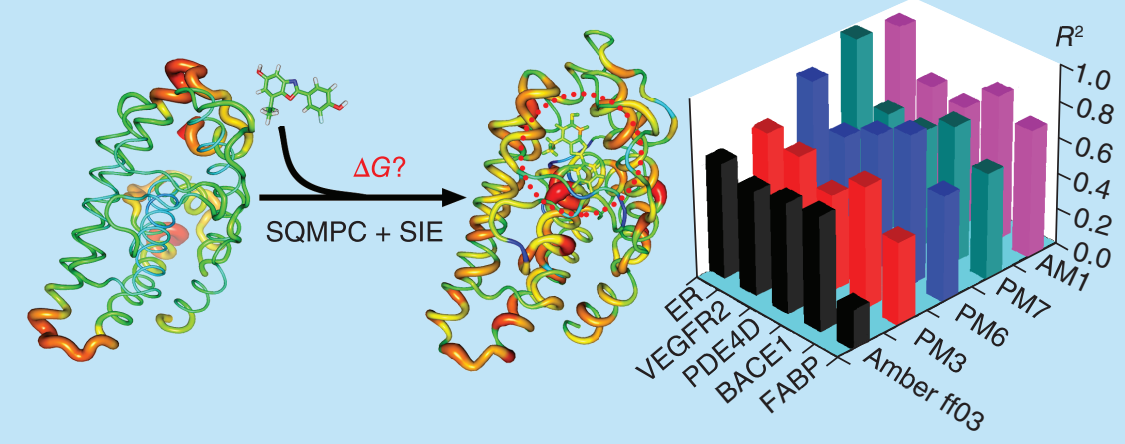

First draft submitted: 31 May 2018; Accepted for publication: 30 November 2018; Published online: 25 February 2019

Keywords: atomic charge $\bullet$ binding-free energy $\bullet$ electrostatic polarization $\bullet$ semiempirical quantum mechanics • solvated interaction energy

Many theoretical methods for studying protein-ligand interaction share a common challenge to accurately calculate the binding-free energy $(\Delta G)$ between protein and ligand, which impedes their reliability and wide application [14]. Strategies of calculating ligand-binding affinities have therefore received increasing attention over decades. For 
example, free energy perturbation [5] and thermodynamic integration [6] approaches are rigorous in calculating $\Delta G$ but computationally very expensive. To improve the efficiency of $\Delta G$ prediction, plentiful methods combined with molecular dynamics (MD) simulation have been developed, including linear interaction energy (LIE) [7,8] as well as molecular mechanics (MM)/Poisson-Boltzmann (or generalized Born) surface area (MM/PBSA or MM/GBSA) [9,10]. Particularly, MM/PB(GB)SA approach has been widely used in $\Delta G$ calculation due to its high time-efficiency and tractability $[1,11]$. However, the overall accuracy of $M M / P B(G B) S A$ approach is sensitive to many factors, including selected portion of MD trajectory, force field [12], partial atomic charge model for ligand [12] and, especially, electrostatic interaction and polarization $[13,14]$.

Solvated interaction energy (SIE) [15] approach provides an effective tool to estimate $\Delta G$. It is a kind of parameterized function including force field and solvation terms, which have been calibrated from a set of 99 protein-ligand complexes. It has been originally designed and recently widely applied to score binding affinity. SIE is computationally efficient for $\Delta G$ calculation [16]. For example, Cui et al. showed the advantage of SIE over MM/GBSA in calculating absolute $\Delta G$ in the simulation of Ras-Raf and Ras-RalGDS [17]. Additionally, SIE approach has been ranked as one of the best scoring functions in several rounds of Community Structure-Activity Resource (CSAR) and Statistical Assessment of the Modeling of Proteins and Ligands (SAMPL) challenges [18-21]. However, the performance of SIE approach on achieving accurate $\Delta G$ prediction is still far from perfect, which might be related to its usage of Amber force field that cannot tackle electrostatic polarization, an indispensable term for predicting $\Delta G$ accurately [22-26].

Therefore, great efforts have recently been made to accurately deal with electrostatic interaction for accurate $\Delta G$ prediction, such as polarizable force fields atomic multipole optimized energetics for biomolecular applications (AMOEBA) [27], sum of interactions between fragments ab initio computed (SIBFA) [28], quantum mechanical polarizable force field (QMPFF) $[29,30]$ and so on. For instance, Jiao et al. showed the critical role of polarizability in pyridine-trypsin binding process using AMOEBA force field [31]. However, the polarization interaction is very difficult to be parameterized $[27,32,33]$, which deters its further wide application. Cho et al. showed that the consideration of polarization by ligand charge recomputation with quantum mechanics/MM (QM/MM) technique increased the robustness and accuracy for molecular docking [26]. In addition, molecular fractionation with conjugate caps method proposed by Zhang et al. could accurately calculate the interaction between protein and another molecule, which took advantages from decomposing protein molecule into amino acid-based fragments [34,35]. Other well-known fragmentation soft-scaling methods, such as kernel energy method, have also been proved powerful for predicting $\Delta G$ of large biological molecules [36-40]. Within this context, we previously developed a method that utilized QM/MM method to calculate the partial atomic charge of protein-ligand complex, named QMPC, which could significantly improve the correlation between computationally predicted and experimental $\Delta G$ [41]. This improvement should be mainly attributed to the precise partial atomic charge of protein-ligand complex calculated with QMPC. For example, the calculated $\Delta G$ of streptavidin and its mutants binding to biotin showed great correlation coefficient $\left(R^{2}\right)$ with experimental data using MM/GBSA coupled with QMPC $\left(R^{2}=0.92\right)$, while the $R^{2}$ decreased to 0.47 by using conventional Amber ff03 charge [41]. Despite the verification of QMPC in accurately calculating $\Delta G$, there is still room for further improvement of this method, such as huge computational resources required for DFT-B3LYP/6-31G* calculation for the partial atomic charge of protein, the accuracy of MM/GBSA approach and its extensibility.

It has been often reported that the accuracy of semiempirical quantum mechanical (SQM) method for noncovalent interaction is comparable to dispersion-corrected density functional theory, while its computational time could be reduced by two to three orders of magnitude [42,43]. For example, our previous research showed that the accuracy of PM6 QM/MM MD simulation is comparable to that of QM(B3LYP)/MM MD simulation in treating hydrogen bonding and polar interactions [44]. Hazai et al. also found that protein-ligand docking could be improved using the partial atomic charge obtained by PM6 [45]. All these results showed that SQM methods might be a good choice for accurately dealing with the electrostatic polarization.

Accordingly, the efficiency and accuracy of $\Delta G$ prediction by SIE or MM/GBSA approach might be further improved if SQM partial atomic charge is incorporated. Encouraged by this idea, a new method for calculating atomic charge of both protein and ligand with SQM/MM approach, named SQMPC, was developed and its efficiency, accuracy and robustness were evaluated with 50 various protein-ligand systems in this study. The proteins are estrogen receptor (ER), fatty acid binding protein (FABP), $\beta$-site amyloid precursor protein cleaving enzyme 1 (BACE1), VEGFR2 and phosphodiesterase 4D (PDE4D). The two quantum chemistry methods, namely, B3LYP/6-31G* and Hartree-Fock (HF)/6-31G*, and four different SQMs, namely, AM1 [46], PM3 [47,48], PM6 [49] 
and PM7 [50] were evaluated. Our results revealed that the performance of PM7 and AM1 is comparable to B3LYP and is better than HF in ER system. Additionally, we also found that longer MD simulation is not always necessary to accomplish better $\Delta G$ prediction. Therefore, the use of SQM methods of AM1 or PM7 to calculate the atomic partial charge of protein is a highly efficient, accurate and extensible approach to improving the accuracy of $\Delta G$ prediction with SIE or MM/GBSA approach.

\section{Methods}

\section{Preparation of complexes}

SQMPC calculation was applied on five different protein systems, namely, ER, FABP, BACE1, VEGFR2 and PDE4D. Ten different ligands for each protein were selected by taking into account both their structural diversity and binding affinity. 48 protein-ligand complexes' structures were directly downloaded from Protein Data Bank (PDB). The protonation state of protein residues was predicted by $H++$ web server [51] (refer to PDB files for each protein in Supplementary Information). As no protein-ligand crystal structures were available in PDB, the initial structures of 3OMQ_CH3 and 3OMO_CH3 systems were derived by substituting fluorine atoms of ligand in $3 \mathrm{OMQ}$ with hydrogen atoms, and substituting the hydroxyl group of ligand in 3OMO with methoxyl group, respectively.

All the protein-ligand complexes were then used as initial models for MD simulation (Supplementary Table 1). Each system was neutralized by placing a rational number of counter ions of $\mathrm{Na}^{+}$or $\mathrm{Cl}^{-}$in grids and solvated into a periodic TIP3P water box, extending $9 \AA$ from the solute. General Amber force field [52] was applied to model ligands. Amber ff03 force filed [53] was used to parameterize proteins since it produces better $\Delta G$ predictions than others (ff99, ff99SB, ff99SB-ILDN, ff12SB) by using both MM/GBSA and MM/PBSA approaches with short timescale MD simulations [12]. Geometry optimization and electrostatic potential calculation of ligand were performed with continuum solvent model (conductor-like polarizable continuum model, $\varepsilon=4$, atomic radii $=$ universal force field) at B3LYP/6-31G* level with Gaussian09, followed by restrained electrostatic potential derived charge calculation of ligand by program antechamber [54]. Missing loop of protein was built by program Modeller (version 7).

\section{QMPC/SQMPC calculation}

Similar protocol described in our previous work [41] was applied to calculate the partial atomic charge of each atom in protein-ligand complex. In brief, a protein with $\mathrm{N}$ residues was decomposed into $\mathrm{N}$ QM/MM systems, each of which was composed of three parts: a core area that was composed of the atoms of the $\mathrm{n}^{\text {th }}$ residue and was calculated with (S)QM methods for electron density; a buffer area that was composed of the two neighbor residues of the $\mathrm{n}^{\text {th }}$ residue and was also calculated with (S)QM methods for electron density; an MM area that was composed of all the remaining atoms of the system. The $\mathrm{n}^{\text {th }}(S)$ QM area was created by the truncation at the $\mathrm{n}-2^{\text {th }}$ and $\mathrm{n}+1^{\text {th }}$ peptide bonds and capping with hydrogen atoms [41]. For the electron density calculation of ligand, the atoms of ligand were treated as (S)QM atoms with the remaining atoms of system treated as MM area. For QMPC, Merz-Kollman charge distribution scheme was used for electron density calculation by our own $n$-layered integrated molecular orbitaland molecular mechanics $(\mathrm{ONIOM})$ scheme $\left(\mathrm{QM}=\mathrm{HF} / 6-31 \mathrm{G}^{*}\right.$ or B3LYP/6-31G*; $\mathrm{MM}=$ Amber ff03) with Gaussian09 [55-58]. To mimic solvent effect, we placed the surface charge calculated by Delphi program into ONIOM calculation as additional background charge [56]. The QMPC was obtained by restrained electrostatic potential program based on the electron density calculated in ONIOM calculation $[57,58]$. The SQMPC in format of Coulson charge was calculated by SQM/MM using MOPAC2016 program [59,60], which was performed in the same way as the Gaussian09 in fragment protocol. The SQMPC was calculated with four different SQM methods, namely, AM1, PM3, PM6 and PM7 (referred to as SQMPC $\mathrm{AM}_{1}, \mathrm{SQMPC}_{\mathrm{PM} 3}, \mathrm{SQMPC}_{\mathrm{PM} 6}$ and SQMPC $\mathrm{PM7}$, respectively, hereinafter). The calculated (S)QMPC was used to replace the initial Amber ff03 charge for the next (S)QMPC calculation until it reached convergence.

\section{Molecular dynamics simulation}

Before (S)QMPC calculation, each simulation system was initially minimized with the steepest descent minimization of 5000 steps and a conjugate gradient minimization of 5000 steps, by freezing heavy atoms of protein-ligand complex $\left(1 \mathrm{kcal} / \mathrm{mol} / \AA^{2}\right)$. Then, each system was heated to $300 \mathrm{~K}$ gradually within $0.2 \mathrm{~ns}$. This step was then followed by another $0.1 \mathrm{~ns}$ equilibration at $300 \mathrm{~K}$ in the constant-pressure, constant-temperature ensemble (NPT). After that, (S)QMPC was calculated and used for both MD simulation and $\Delta G$ calculation. Finally, we performed 
5 ns MD simulation on each system at $300 \mathrm{~K}$ using (S)QMPC, as well as conventional Amber ff03 charge for comparison, respectively. The minimization, heating-up and equilibrium processes were performed with sander program of Amber 14. The 5 ns production run was performed with pmemd.cuda (GPU Tesla K40), using Langevin dynamics to control temperature. The SHAKE algorithm [61] was applied to fix bonds involving hydrogen atoms. The particle mesh Ewald method was used to deal with long-range electrostatic interactions [62].

\section{Binding-free energy calculation}

Based on the 5 ns MD simulation trajectory, $\triangle G$ was calculated with SIE and MM/GBSA approaches. Regarding MM/GBSA approach, it belongs to physics-based scoring functions, which depend on some physical properties of the system, such as dielectric constants for solvent and solute and atomic Born radii. The $\Delta G$ was calculated according to Equation (1),

$$
\Delta G=\Delta H-T \Delta S=\Delta E_{\text {ele }}+\Delta E_{V D W}+\Delta G_{g b}+\Delta G_{n p}-T \Delta S
$$

(Equation 1)

where $\Delta E_{\text {ele }}$ and $\Delta E_{\mathrm{VDW}}$ represent electrostatic and van der Waals energy terms, respectively. $\Delta G_{\mathrm{gb}}$ and $\Delta G_{\mathrm{np}}$ represent polar and nonpolar solvation-free energies, respectively. Conformational entropy $(T \Delta S)$ was calculated by nmode of Amber14. The dielectric constants for solvent and solute were set to 80.0 and 1.0, respectively, and Onufriev, Bashford, Case $(\mathrm{OBC})$ solvation model (igb $=5$ and PBradii $=5)$ [63] was used in this study. The $\Delta G_{\mathrm{np}}$ was calculated in Equation (2),

$$
\Delta G_{n p}=\gamma S A S A+\beta
$$

(Equation 2)

where the solvent accessible surface area (SASA) was calculated using program mach Stichprobe mit Selektion (MSMS) [64]. The values for $\gamma$ and $\beta$ were set to be $0.0072 \mathrm{kcal} / \mathrm{mol} / \AA^{2}$ and $0.0 \mathrm{kcal} / \mathrm{mol}$, respectively.

Regarding SIE approach [15], it belongs to end point force field-based scoring functions, of which physical parameters are optimized from a dataset based on force field. The $\Delta G$ was calculated based on Equation (3),

$$
\Delta G_{\text {bind }}=\alpha\left[E_{c}\left(D_{\text {in }}\right)+\Delta G_{\text {bind }}^{R}\left(\rho, D_{\text {in }}\right)+\Delta E_{V D W}+\gamma \Delta M S A(\rho)\right]+C
$$

where $E_{\mathrm{C}}$ and $E_{\mathrm{VDW}}$ represent the sum of intermolecular Coulomb and van der Waals interaction energies, respectively. $\Delta G^{\mathrm{R}}$ bind and $\triangle M S A$ are the changes of reaction field energy and molecular surface area (MSA) upon ligand binding, respectively. $\Delta G$ Rind was calculated by using boundary element method $[65,66]$ to solve Poisson equation, and MSA was determined by a variable-radius solvent probe [67]. Default values of the five parameters in SIE approach $\left(\alpha=0.1048, D_{\text {in }}=2.25, \rho=1.1, \gamma=0.0129 \mathrm{kcal} / \mathrm{mol} / \AA^{2}\right.$, and $\left.\mathrm{C}=-2.89 \mathrm{kcal} / \mathrm{mol}\right)$ were used in this study.

\section{Results \& discussions}

\section{Performance of QMPC \& SQMPC on ER ligand systems}

As previously described [41], QMPC could tackle electrostatic polarization effect properly by giving more precise atomic charge of protein system compared with conventional Amber ff03 force field, but it is computationally expensive due to B3LYP/6-31 G* calculation. As expected, all the four different SQMPC methods $(1.5 \mathrm{~h}$ per system in general) consumed nearly 20 -fold less computational resources than the $\mathrm{QMPC}_{\mathrm{B} 3 \mathrm{LYP} / 6-31 \mathrm{G}^{*}}$ or $\mathrm{QMPC}_{\mathrm{HF} / 6-31 \mathrm{G}^{*}}$ methods (30 h per system in general) for ER-ligand complexes, demonstrating that SQMPC method is much more computationally efficient than QMPC for calculating partial atomic charge of protein-ligand complex. Meanwhile, root-mean-square deviation (RMSD) of heavy atoms in ten different systems along $5 \mathrm{~ns} \mathrm{MD}$ simulation was analyzed (Supplementary Figure 1). The results showed that the RMSD to mean value after $0.5 \mathrm{~ns}$ is within $1.0 \AA$, indicating that MD trajectories after $0.5 \mathrm{~ns}$ could be used for $\Delta G$ calculation. In addition, we found that $\mathrm{QMPC}_{\mathrm{B} 3 \mathrm{LY}}$ produces the lowest $\mathrm{RMSD}$, and $\mathrm{SQMPC}_{\mathrm{AM} 1}$ obtains the most stable trajectory over the $0.5-5$ ns MD simulation, while Amber ff03 obtains the most unstable trajectory, indicating that (S)QMPC could improve the stability of MD simulation (Supplementary Table 2).

Figure 1 depicted the $R^{2}$ between experimentally measured and the theoretically predicted $\Delta G$ using different approaches. The $R^{2}$ of SIE-B3LYP, SIE-HF, SIE-AM1, SIE-PM3, SIE-PM6 and SIE-PM7 are $0.92 \pm 0.01$, 
Figure 1. Correlation coefficients of QMPC (a method that utilized quantum mechanics/molecular mechanics method to calculate the partial atomic charge of protein-ligand complex; B3LYP \& HF), SMQPC (AM1, PM3, PM6 and PM7) and Amber ff03 charge. Binding free energies were calculated by solvated interaction energy and molecular mechanics/generalized Born surface area approaches based on 0.5-5 ns molecular dynamics simulation trajectory.

MM/GBSA: Molecular mechanics/generalized Born surface area; SIE: Solvated interaction energy.

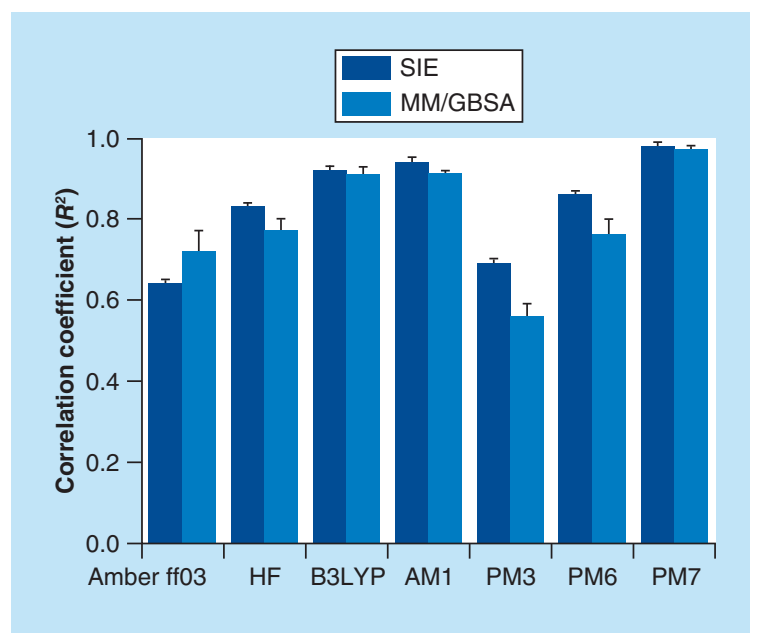

$0.83 \pm 0.01,0.94 \pm 0.01,0.69 \pm 0.01,0.86 \pm 0.01$ and $0.98 \pm 0.01$, respectively, where SIE-B3LYP stands for the method of calculating $\Delta G$ by SIE approach with atomic charge of $\mathrm{QMPC}_{\mathrm{B} 3 \mathrm{LYP}}$, and other designations for the rest methods are in a similar way. Noticeably, all the aforementioned $R^{2}$ are much better than that of SIE-ff03 $\left(R^{2}=0.64 \pm 0.01\right)$, indicating that (S)QMPCs could lead to significant improvement in $\Delta G$ prediction. Similarly, the $R^{2}$ of MM/GBSA-B3LYP, MM/GBSA-HF, MM/GBSA-AM1, MM/GBSA-PM6 and MM/GBSA-PM7 are $0.90 \pm 0.02,0.76 \pm 0.03,0.88 \pm 0.01,0.77 \pm 0.04$ and $0.98 \pm 0.01$, respectively, which are better than that of MM/GBSA-ff03 ( $\left.R^{2}=0.74 \pm 0.05\right)$. Overall, $R^{2}$ of $\Delta G$ prediction is greatly improved for trajectories with (S)QMPC. However, as noted above, SQM methods consume much less computational resources than B3LYP and HF methods. Additionally, among different SQMPCs, the SQMPC $\mathrm{PM}_{7}$ and $\mathrm{SQMPC}_{\mathrm{AM} 1}$ methods achieve similar or even higher $R^{2}$ than that by QMPC methods with SIE or MM/GBSA approach. The mean unsigned errors of $\Delta G$ calculated by SIE-SQMPCs are better than that of SIE-ff03 (Supplementary Table 3). It was also noticeable that the mean unsigned errors of different SIE-SQMPC approaches are stable, but that are very sensitive to different MM/GBSA-SQMPC approaches. In addition, SIE-SQMPC is computationally more efficient than MM/GBSA-SQMPC that is time consuming in entropy calculation (in general, $3 \mathrm{~h}$ with SIE-SQMPC and $50 \mathrm{~h}$ with MM/GBSA-SQMPC per system). Thus, SIE-SQMPC, especially SIE-SQMPC ${ }_{P M 7}$ and SIE-SQMPC AM1 $_{1}$, is a good choice for free energy calculation.

To further investigate the reason why (S)QMPC achieved better results than Amber ff03 charge, individual components of the $\Delta G$ calculated by SIE and MM/GBSA were studied (Table $1 \&$ Supplementary Table 4). The results by SIE approach suggested that the overall electrostatic interaction term, namely $E_{\text {ele }}\left(E_{\text {ele }}=E_{\mathrm{C}}+\Delta G\right.$ bind $)$, is a dominant contribution to $\Delta G$ difference among different approaches. Taking $1 \mathrm{X} 7 \mathrm{~B}$ as an example, comparing with $E_{\mathrm{vdw}}$ difference $(1.12 \mathrm{kcal} / \mathrm{mol})$ between SIE-B3LYP and SIE-ff03 results, the $E_{\text {ele }}$ difference between the two approaches is as much as $-7.72 \mathrm{kcal} / \mathrm{mol}$, indicating that electrostatic interaction plays a more dominant role in $\Delta G$ calculation with different approaches (Table 1). In line with its relatively unsatisfactory $R^{2}$, SIE-PM3 also gives smaller percentage of the $E_{\text {ele }}$ difference than other SIE-SQMPC approaches (Supplementary Figure 2), demonstrating the significance of electrostatic interaction again. Similar to SIE method, the electrostatic interaction $\left(\Delta E_{\mathrm{ele}}+\Delta G_{\mathrm{gb}}\right)$ in MM/GBSA approach was also found to be important in $\Delta G$ calculation.

To compare different (S)QMPC methods directly, two complexes with similar ligands, namely, 1X7B and 1X76, were taken as examples for further analysis (Figure 2). For complex 1X7B, the partial atomic charges of protein calculated by using either the $\mathrm{QMPC}_{\mathrm{B} 3 \mathrm{LYP}}$ or $\mathrm{SQMPC}_{\mathrm{PM} 7}$ methods are quite different from Amber ff03 charge (Figures $2 \mathrm{~A} \& \mathrm{~B}$ ). Similar differences were also found between other (S)QMPC methods (QMPC $\mathrm{HF}_{\mathrm{HF}}$, $\mathrm{SQMPC}_{\mathrm{AM} 1}, \mathrm{SQMPC}_{\mathrm{PM} 3}$ and SQMPC $\mathrm{PM}_{6}$ ) and Amber ff03 (Supplementary Figure 3). In addition, charges obtained by $\mathrm{QMPC}_{\mathrm{B} 3 \mathrm{LYP}}$ and $\mathrm{SQMPC} \mathrm{PM}_{\mathrm{PM}}$ are also slightly different from each other for those residues around ligand (Figure 2C). Furthermore, the partial atomic charges of the residues around the binding pocket of 1X7B (e.g., V98, F26, F93 and H212) calculated by QMPC $_{\mathrm{B} 3 \mathrm{LYP}}$ are quite different from that of $1 \mathrm{X} 76$ (Figure 2D), implying that $\mathrm{QMPC}_{\mathrm{B} 3 \mathrm{LYP}}$ can distinguish different polarization induced by two similar ligands. A similar result was also observed if SQMPC $\mathrm{PM}_{7}$ was applied on the two complexes (Figure 2E), suggesting that both QMPC and SQMPC could deal with polarization effectively. 


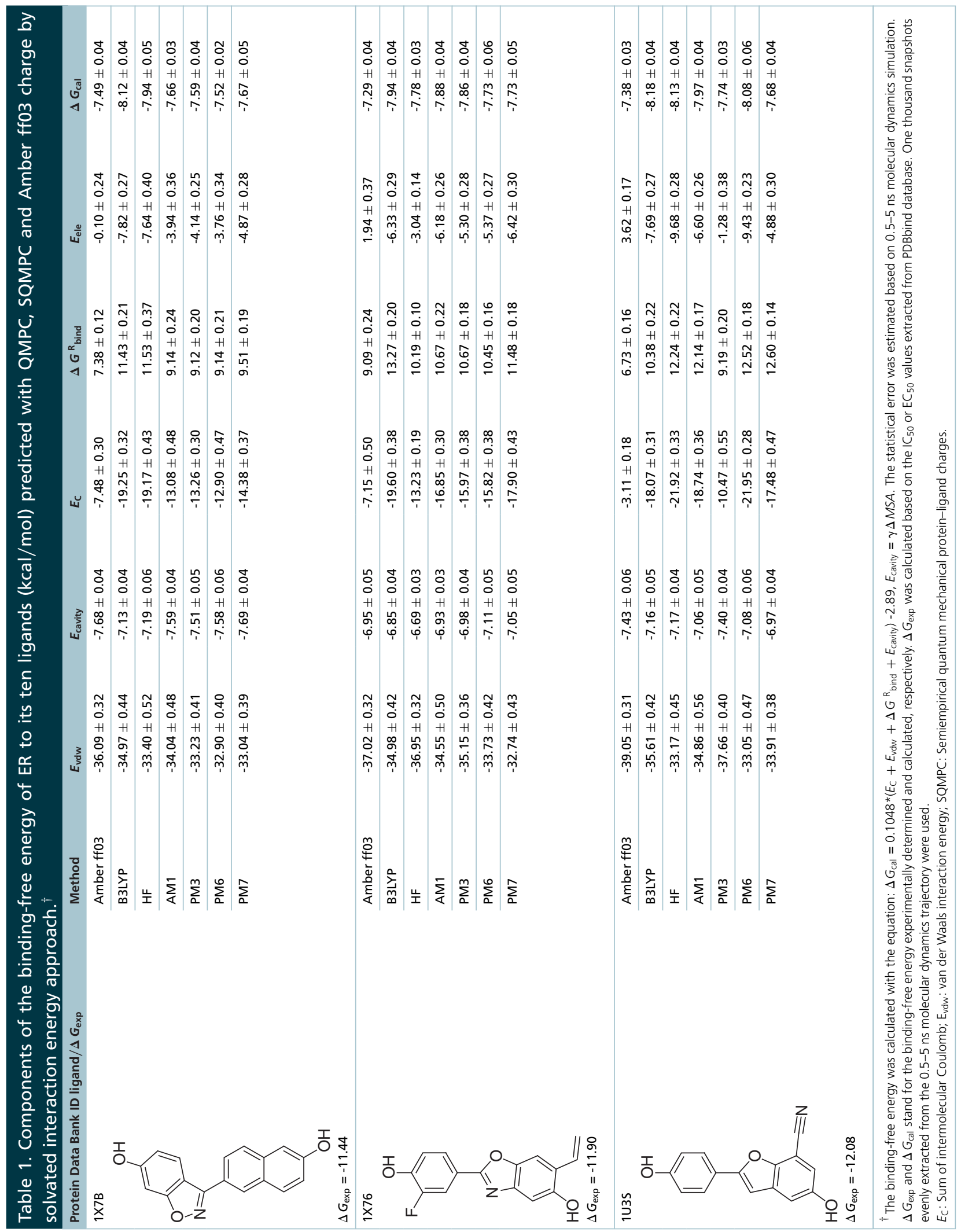




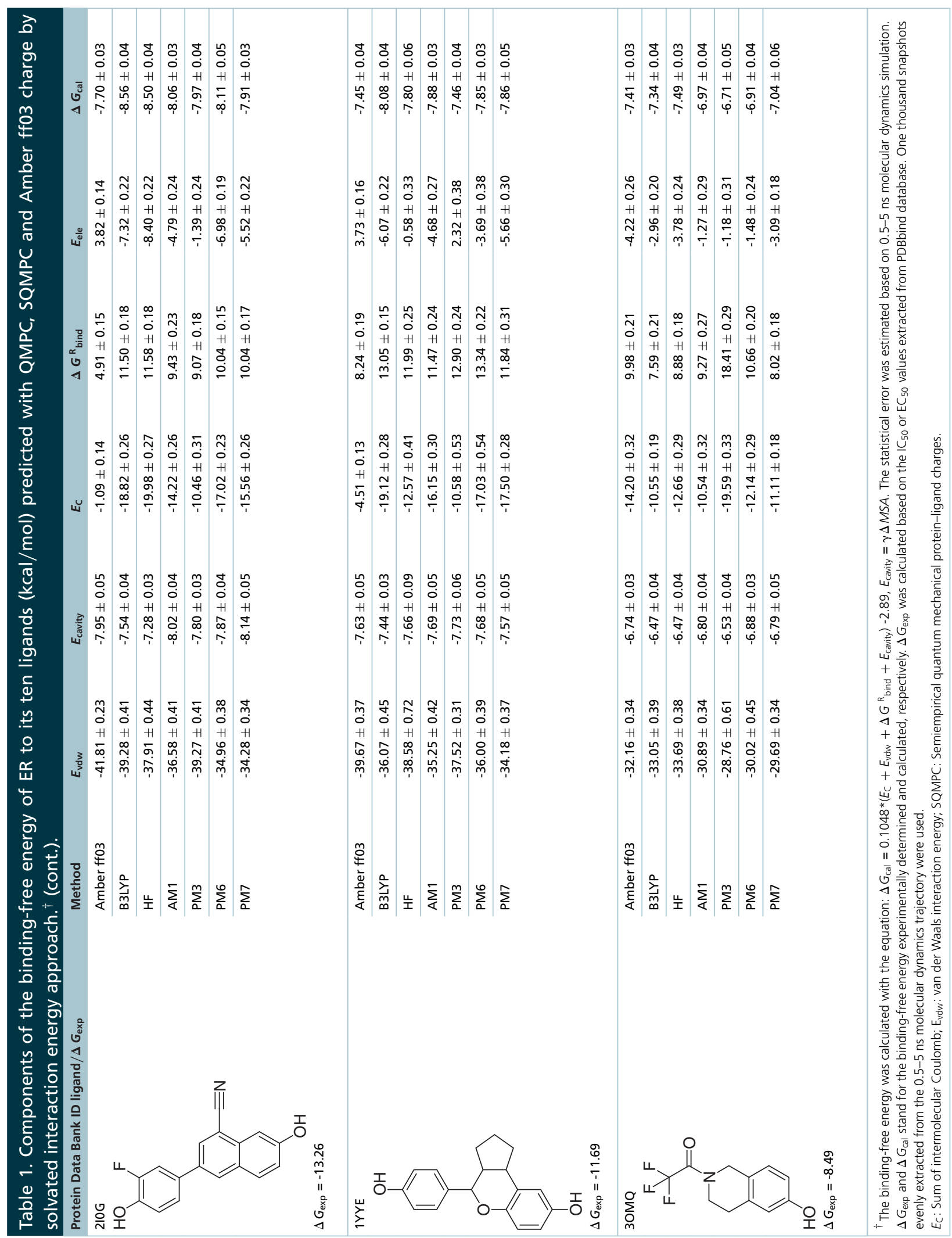




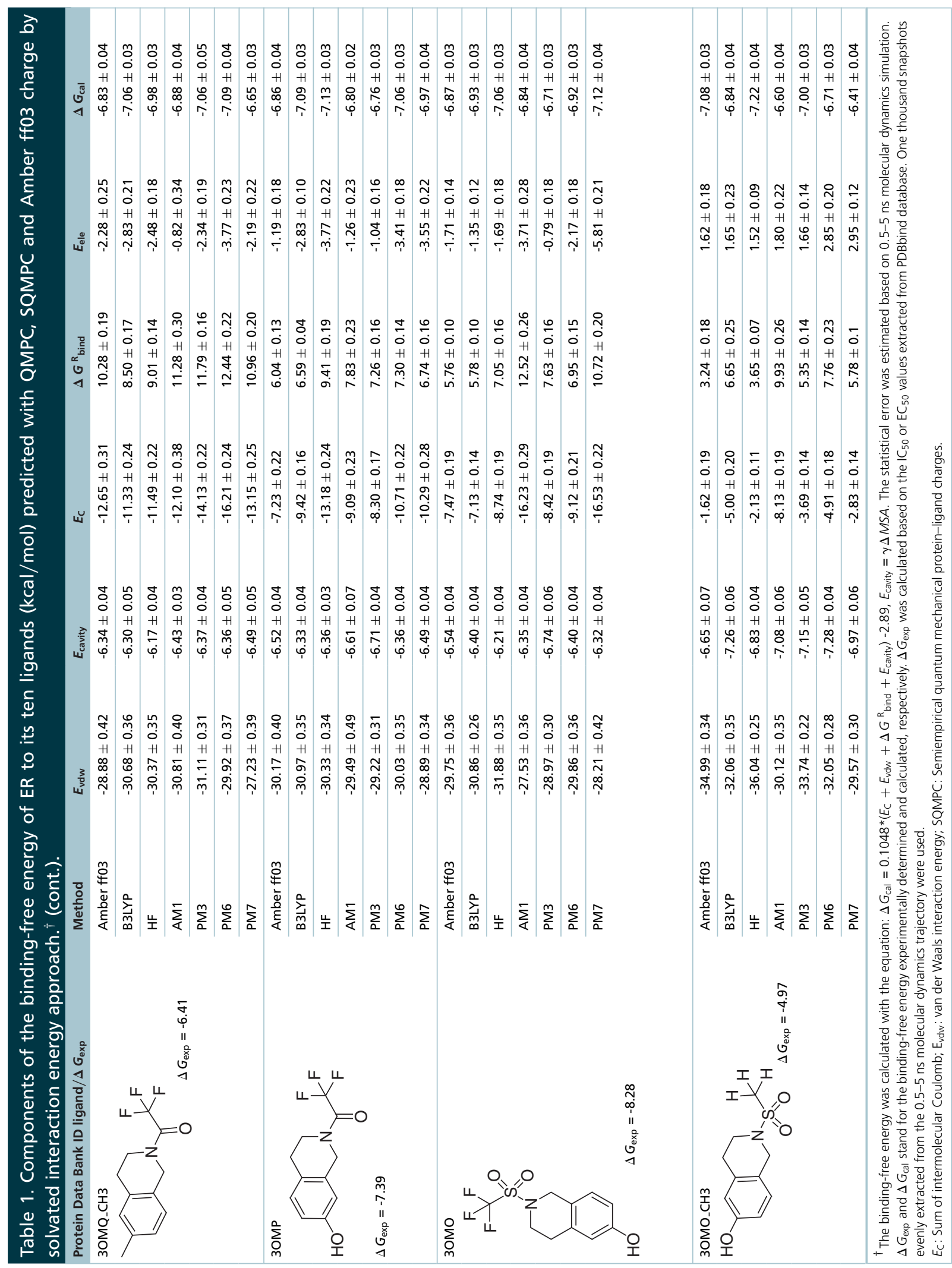




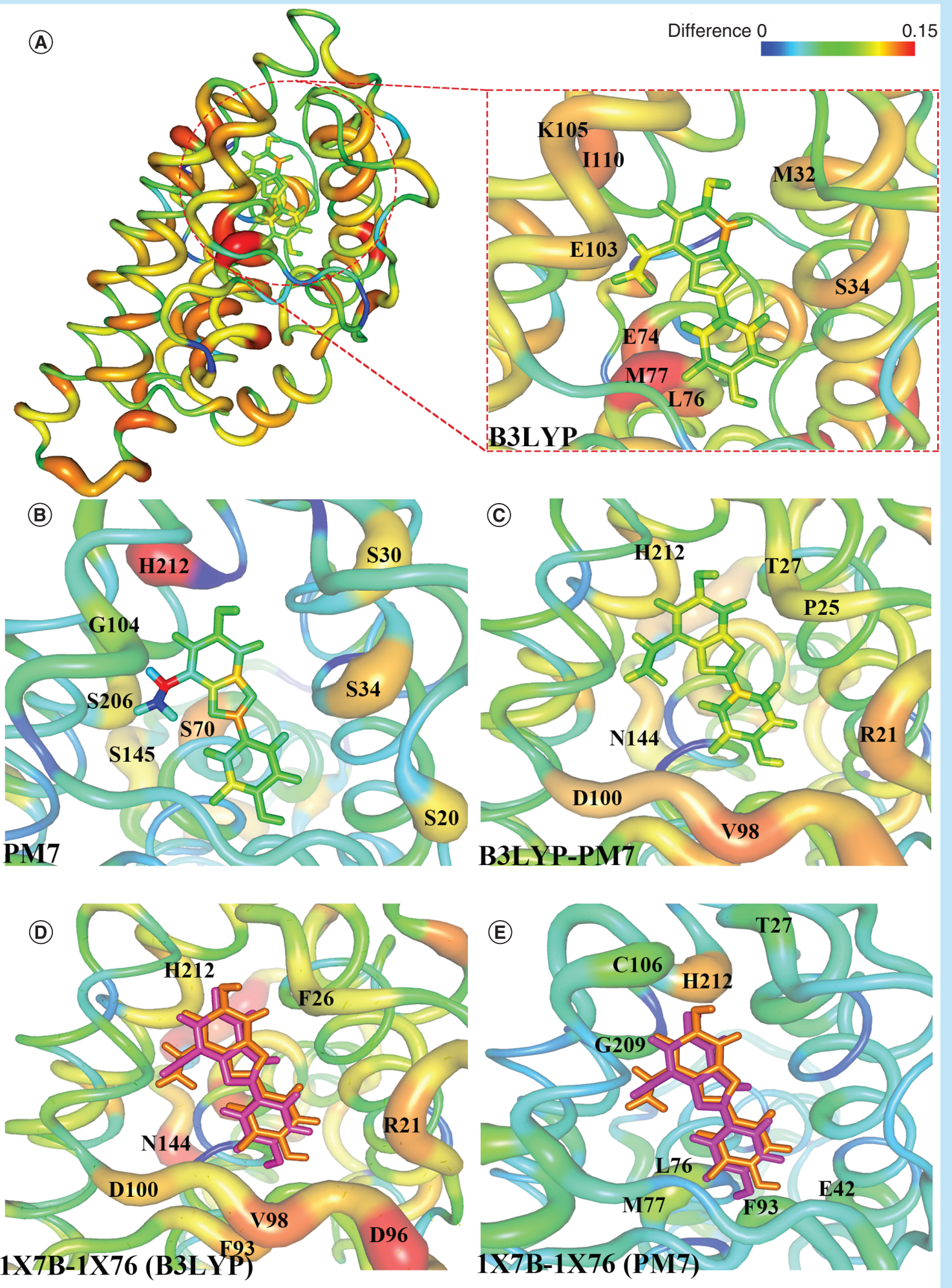

Figure 2. The difference of partial atomic charge calculated by different methods. For $1 \mathrm{X} 7 \mathrm{~B}$, the partial atomic charge difference (A) between Amber ff03 and QMPCB3LYP methods; (B) between Amber ff03 and semiempirical quantum mechanical protein-ligand charges PM7; (C) between QMPCB3LYP and semiempirical quantum mechanical protein-ligand charges PM7, was shown. The difference of protein partial atomic charge with $\mathrm{QMPC}_{\mathrm{B} 3 L \mathrm{LY}}(\mathrm{D})$ between $1 \mathrm{X7B}$ and $1 \mathrm{X76}$, the difference of protein partial atomic charge with QMPC $\mathrm{PM7}_{\mathbf{1}}(\mathrm{E})$ between $1 \mathrm{X7B}$ and $1 \mathrm{X76}$, was shown. The most (least) different partial atomic charge was depicted in bulkier (smaller) size and red (blue), respectively. For (D) and (E), ligands in 1X7B and 1 X76 were colored in orange and pink, respectively. The figure was drawn and colored by the absolute value of difference. 
(A)
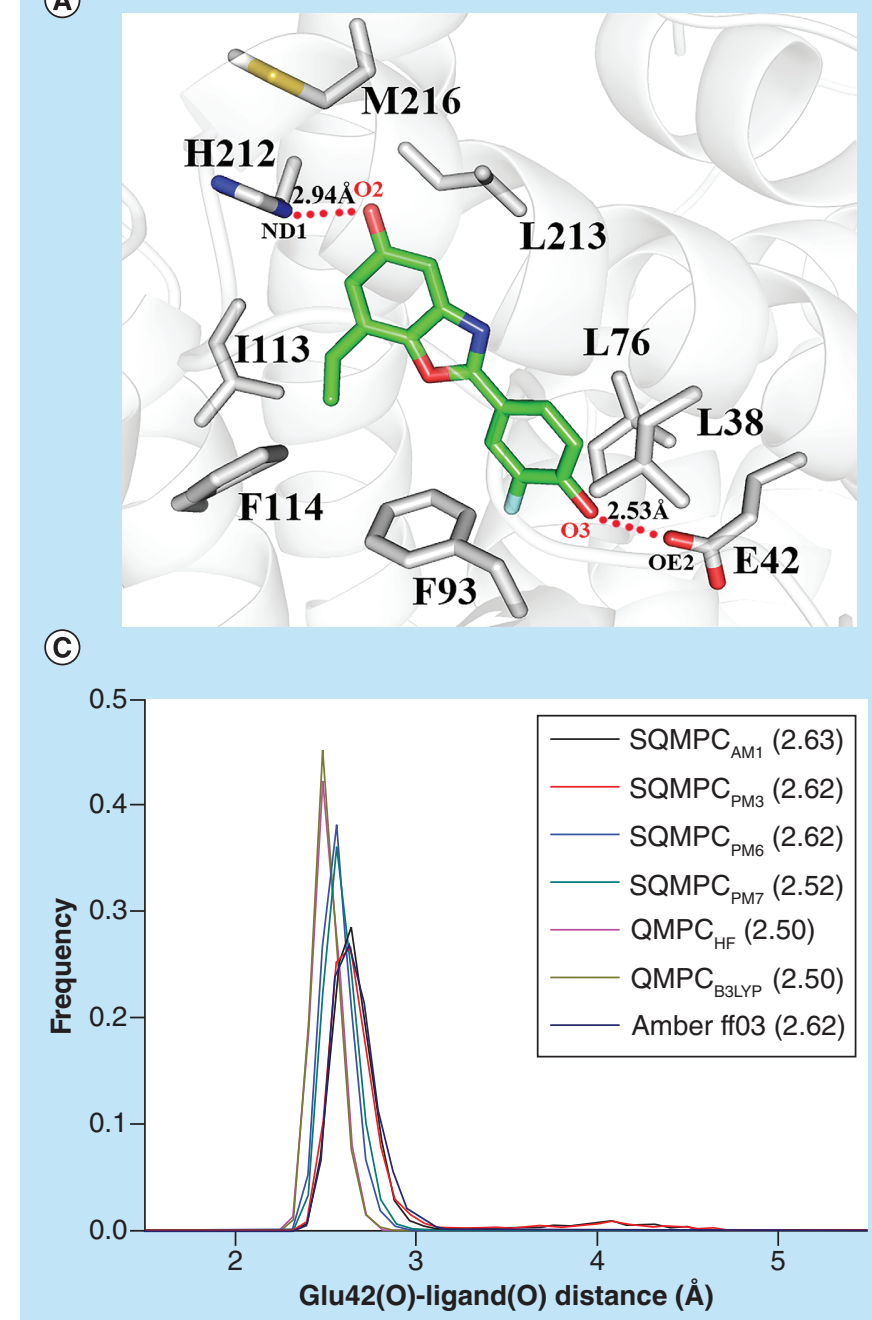

(B)

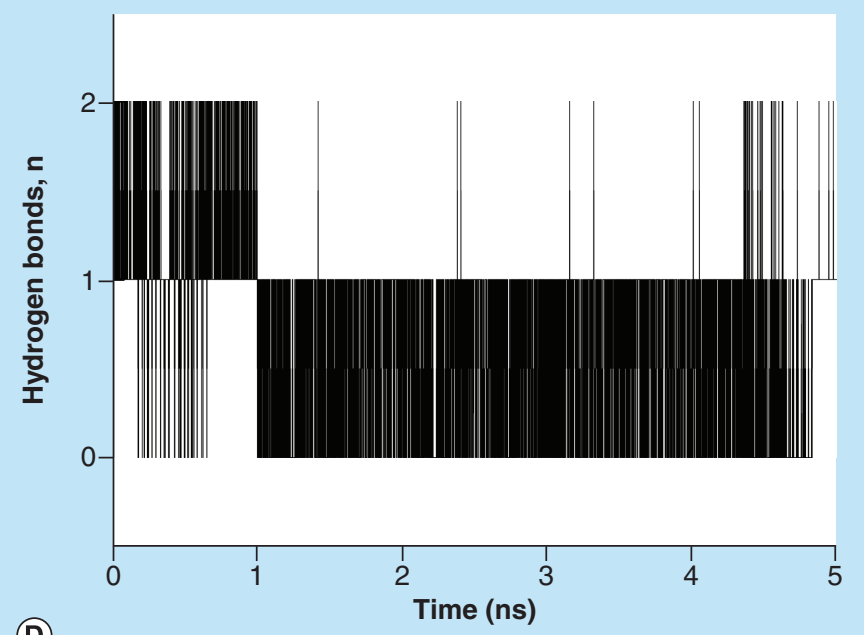

(D)

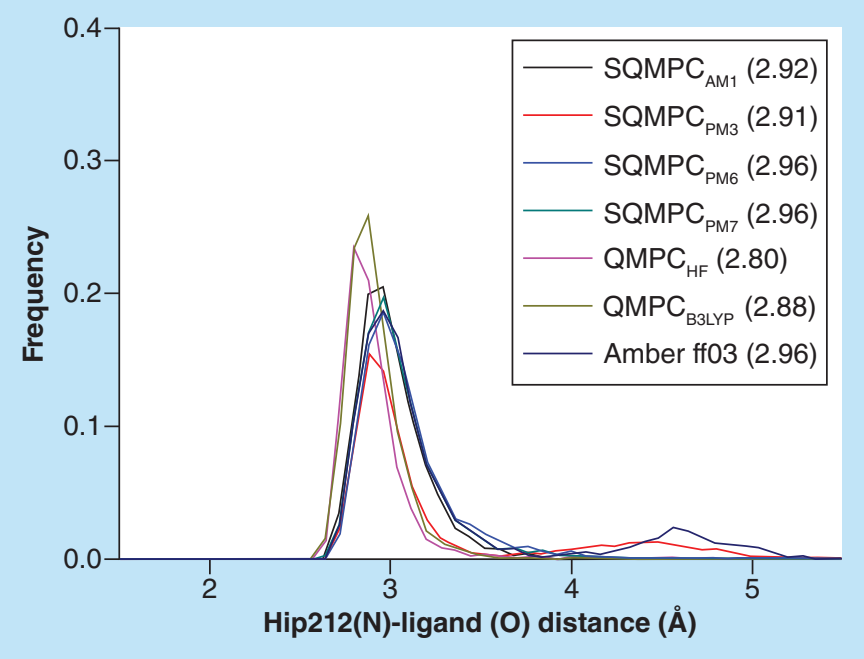

Figure 3. The hydrogen bonds formed during simulation with different methods. (A) X-ray crystal structure of binding pocket in $1 \mathrm{X} 7 \mathrm{~B}$. The residues within binding pocket are labeled. The red dashed line represents the hydrogen bond formed between the ligand and protein. (B) Number of hydrogen bonds during molecular dynamics simulation with SQMPC $\mathrm{PM}_{\mathrm{in}}$ iX7B. The frequency distribution of ND1 (His212)-O2 (ligand) (C) and O3(ligand)-OE2(Glu42) (D) distances along 5 ns molecular dynamics simulation using QMPC, SQMPC and Amber ff03 charge in 1X7B system. The peak distance values are shown in bracket.

SQMPC: Semiempirical quantum mechanics protein-ligand complex.

Hydrogen bond is another key factor closely associated with $\Delta G$ prediction. Accordingly, the program $c p p t r a j$ of Amber14 was used to analyze hydrogen bonds along the $5 \mathrm{~ns}$ MD simulation. Taking 1X7B as an example (Figure 3A), two hydrogen bonds were found between the ligand and the protein during simulations. The first hydrogen bond is formed between the oxygen atom $\mathrm{O} 3$ of ligand and the oxygen atom OE2 of Glu42, the second is formed between $\mathrm{O} 2$ of ligand and ND1 of His212. It was found that the number of these two hydrogen bonds continuously fluctuated, while at least one hydrogen bond was observed during MD simulation with SQMPC $\mathrm{PM}_{7}$ (Figure 3B). The frequency versus hydrogen bond length was analyzed (Figure 3C \& D). Although the peak distance of the first hydrogen bond calculated with $\mathrm{SQMPC}_{\mathrm{PM}}$, $\mathrm{SQMPC}_{\mathrm{PM} 7}, \mathrm{QMPC}_{\mathrm{HF}}$ and $\mathrm{QMPC}_{\mathrm{B} 3 \mathrm{LYP}}(2.50-2.52 \AA)$ is little shorter than that with $\mathrm{SQMPC}_{\mathrm{PM}}, \mathrm{SQMPC}_{\mathrm{AM} 1}$ and Amber ff03 (2.62-2.63 $\AA$ ) (Figure 3C), all the distances demonstrated that the first hydrogen bond is quite stable during the simulations. However, the peak distance of the second hydrogen bond (Figure 3D) calculated with all methods ( 2.80 and $2.96 \AA)$ is longer than that of the first hydrogen bond, suggesting that the first hydrogen bond is more stable than the second one, which is in line 
Table 2. Mean value of the hydrogen bond length $(\AA)$ in ER-inhibitor systems in molecular dynamics simulation with QMPC, SQMPC and Amber ff03 charge. ${ }^{\dagger}$

\begin{tabular}{|c|c|c|c|c|c|c|c|c|c|}
\hline System & Hydrogen bond & X-ray & AM1 & PM3 & PM6 & PM7 & HF & B3LYP & Amber ff03 \\
\hline \multirow[t]{2}{*}{$1 \times 7 B$} & Glu42(O)-LBD(O) & 2.53 & $2.74 \pm 0.37$ & $2.74 \pm 0.37$ & $2.58 \pm 0.09$ & $2.56 \pm 0.09$ & $2.50 \pm 0.08$ & $2.50 \pm 0.08$ & $2.67 \pm 0.13$ \\
\hline & Hip212(N)-LBD(O) & 2.94 & $3.02 \pm 0.22$ & $3.64 \pm 0.93$ & $3.05 \pm 0.27$ & $3.09 \pm 0.28$ & $3.11 \pm 0.57$ & $2.91 \pm 0.19$ & $3.24 \pm 0.79$ \\
\hline $1 \times 76$ & Hip212(N)-LBD(O) & 3.28 & $3.01 \pm 0.20$ & $4.31 \pm 1.12$ & $3.04 \pm 0.23$ & $3.29 \pm 0.49$ & $4.21 \pm 0.56$ & $3.28 \pm 0.77$ & $4.20 \pm 0.61$ \\
\hline \multirow[t]{3}{*}{$1 \mathrm{US}$} & $\operatorname{Hip} 212(\mathrm{~N})-\mathrm{LBD}(\mathrm{O})$ & 2.91 & $2.96 \pm 0.22$ & $4.08 \pm 0.89$ & $3.36 \pm 0.77$ & $4.60 \pm 0.75$ & $3.29 \pm 0.16$ & $4.52 \pm 1.64$ & $4.62 \pm 1.31$ \\
\hline & Glu42(O)-LBD(O) & 2.61 & $2.62 \pm 0.21$ & $3.10 \pm 1.16$ & $2.57 \pm 0.09$ & $2.58 \pm 0.09$ & $2.48 \pm 0.07$ & $2.50 \pm 0.08$ & $5.30 \pm 0.85$ \\
\hline & $\operatorname{Arg} 83(\mathrm{~N})-\mathrm{LBD}(\mathrm{O})$ & 3.40 & $4.25 \pm 0.44$ & $3.54 \pm 0.50$ & $4.23 \pm 0.37$ & $4.18 \pm 0.32$ & $3.43 \pm 0.27$ & $3.44 \pm 0.27$ & $4.31 \pm 0.47$ \\
\hline \multirow[t]{3}{*}{$210 \mathrm{G}$} & $\operatorname{Hip} 212(\mathrm{~N})-\mathrm{LBD}(\mathrm{O})$ & 3.42 & $2.98 \pm 0.17$ & $3.05 \pm 0.24$ & $3.00 \pm 0.18$ & $2.98 \pm 0.16$ & $2.88 \pm 0.13$ & $2.90 \pm 0.16$ & $5.28 \pm 0.84$ \\
\hline & Glu42(O)-LBD(O) & 2.46 & $2.63 \pm 0.10$ & $3.83 \pm 0.87$ & $2.61 \pm 0.10$ & $2.64 \pm 0.11$ & $2.49 \pm 0.08$ & $2.52 \pm 0.08$ & $5.64 \pm 1.12$ \\
\hline & $\operatorname{Arg} 83(\mathrm{~N})-\mathrm{LBD}(\mathrm{O})$ & 2.99 & $3.61 \pm 0.42$ & $3.47 \pm 0.37$ & $3.50 \pm 0.28$ & $3.78 \pm 0.36$ & $3.43 \pm 0.24$ & $3.51 \pm 0.52$ & $3.45 \pm 0.50$ \\
\hline \multirow[t]{2}{*}{ 1YYE } & $\operatorname{Hip} 212(\mathrm{~N})-\mathrm{LBD}(\mathrm{O})$ & 3.18 & $3.10 \pm 0.39$ & $5.56 \pm 1.11$ & $5.68 \pm 0.49$ & $5.20 \pm 1.17$ & $3.03 \pm 0.38$ & $2.93 \pm 0.15$ & $5.26 \pm 1.17$ \\
\hline & Leu76(O)-LBD(O) & 2.77 & $3.67 \pm 0.38$ & $3.74 \pm 0.46$ & $3.53 \pm 0.28$ & $3.49 \pm 0.27$ & $2.71 \pm 0.17$ & $3.60 \pm 0.28$ & $4.57 \pm 0.86$ \\
\hline 3OMQ & Leu76(O)-LBD(O) & 2.86 & $3.53 \pm 0.30$ & $3.54 \pm 0.24$ & $3.82 \pm 0.29$ & $3.92 \pm 0.27$ & $3.50 \pm 0.30$ & $3.43 \pm 0.56$ & $3.69 \pm 0.34$ \\
\hline 3OMQ_CH3 & Glu42(O)-LBD(O) & 2.88 & $3.49 \pm 0.28$ & $3.52 \pm 0.30$ & $3.69 \pm 0.32$ & $3.65 \pm 0.37$ & $3.67 \pm 0.33$ & $3.26 \pm 0.26$ & $3.74 \pm 0.30$ \\
\hline $30 \mathrm{MP}$ & Glu42(O)-LBD(O) & 2.60 & $2.67 \pm 0.12$ & $2.68 \pm 0.12$ & $2.63 \pm 0.09$ & $2.66 \pm 0.10$ & $2.62 \pm 0.10$ & $2.63 \pm 1.96$ & $2.66 \pm 0.10$ \\
\hline 3омо & Glu42(O)-LBD(O) & 3.03 & $2.59 \pm 0.09$ & $2.65 \pm 0.10$ & $2.59 \pm 0.08$ & $2.53 \pm 0.08$ & $2.54 \pm 0.08$ & $2.62 \pm 0.08$ & $2.63 \pm 0.12$ \\
\hline Mean value & & 2.93 & $3.12 \pm 0.26$ & $3.56 \pm 0.58$ & $3.32 \pm 0.26$ & $3.41 \pm 0.33$ & $3.06 \pm 0.23$ & $3.10 \pm 0.47$ & $4.08 \pm 0.63$ \\
\hline
\end{tabular}

with the length of the two hydrogen bonds in x-ray crystal structure (2.53 and $2.94 \AA$, respectively, Figure $3 \mathrm{~A})$. It was also found that Amber ff03 always produced longer hydrogen bond than other methods and crystal structure, demonstrating that Amber ff03 has a weaker ability to reproduce hydrogen bonds in crystal structure.

Table 2 shows the mean lengths of hydrogen bonds for all ER-ligand complexes during simulations, which are 3.10, 3.06, 3.12, 3.32, 3.41 and 3.56 $\AA$ with the $\mathrm{QMPC}_{\mathrm{B} 3 \mathrm{LYP}}, \mathrm{QMPC}_{\mathrm{HF}}, \mathrm{SQMPC}_{\mathrm{AM} 1}, \mathrm{SQMPC}_{\mathrm{PM} 6}, \mathrm{SQMPC}_{\mathrm{PM} 7}$ and $\mathrm{SQMPC}_{\mathrm{PM} 3}$ charges, respectively, but $4.08 \AA$ with Amber ff03 charge. These results showed once again that hydrogen bond treated with Amber ff03 charge is obviously weaker than that with (S)QMPC for ER-ligand system. Compared with the mean length of hydrogen bonds of x-ray crystal structures (2.93 $\AA$ ), hydrogen bonds treated by the $\mathrm{QMPC}_{\mathrm{B} 3 \mathrm{LYP}}, \mathrm{QMPC}_{\mathrm{HF}}, \mathrm{SQMPC}_{\mathrm{AM} 1}, \mathrm{SQMPC}_{\mathrm{PM}}$ and $\mathrm{SQMPC}_{\mathrm{PM} 7}$ methods are more stable than that treated by the $\mathrm{SQMPC}_{\mathrm{PM} 3}$. Considering the better $R^{2}$ of ER-ligand complexes with either QMPC or SQMPC method than that with Amber ff03 charge, it could be concluded that (S)QMPC method could reproduce the stability of hydrogen bonds in ER-ligand complexes, which makes a substantial contribution to the binding affinity.

All the above results and discussions suggested that QMPC and most of SQMPC charges could achieve better $\Delta G$ prediction than Amber ff03 charge by either SIE or MM/GBSA approaches, but its expansibility needs to be further explored.

\section{Expansibility on diverse target proteins}

To investigate whether the accuracy of MMGBSA-SQMPC and SIE-SQMPC approaches is independent of protein systems, 40 additional protein-ligand complexes were studied (FABP, BACE1, PDE4D and VEGFR2, with ten different ligands for each protein). MD simulation and binding affinity calculation were performed in the same way as ER-ligand complexes. Similarly, we compared the $R^{2}$ between the predicted and experimental $\Delta G$ to evaluate the performance of these different SQMPC methods on different protein systems. As shown in Figure 4, almost all SQMPC methods achieve better correlation $\left(R^{2}\right)$ for all the additional 40 protein-ligand systems than that by Amber ff03 charge except SQMPC ${ }_{\mathrm{PM} 3}$ (refer to Supplementary Tables $4 \& 5$ for the calculated $\Delta G$ components; Supplementary Figures $4 \& 5$ for the scatter plots of $R^{2}$ ).

For FABP complexes, SIE-AM1 performs especially well with $R^{2}=0.71 \pm 0.02$ (Figure 4A), which is substantially better than that by SIE-ff03 $\left(R^{2}=0.22 \pm 0.02\right)$. SIE-PM7 and SIE-PM6 also have good performance with $R^{2}=0.59 \pm 0.02$. Similarly, the results of MM/GBSA-SQMPCs are better than that of MM/GBSA-ff03 $\left(R^{2}=0.25 \pm 0.04\right)$. 

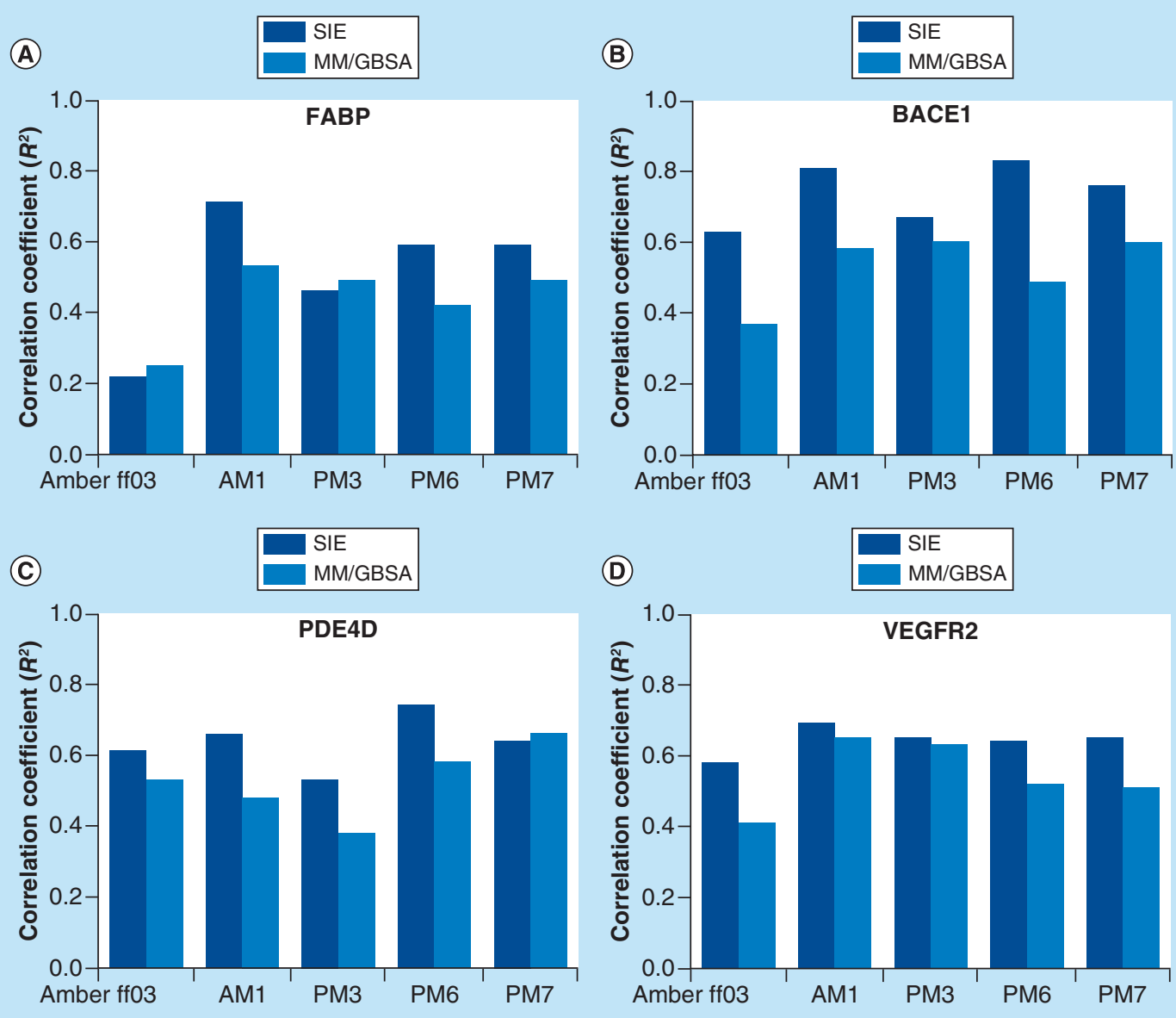

Figure 4. The correlation coefficients between experimentally determined and predicted binding-free energy with solvated interaction energy-SQMPC and molecular mechanics/generalized Born surface area-SQMPC approaches. (A) FABP, (B) BACE1, (C) PDE4D, (D) VEGFR2. All of them were calculated with program sietraj based on $0.5-5 \mathrm{~ns}$ molecular dynamics simulation trajectory. SIE: Solvated interaction energy; MM/GBSA: Molecular mechanics/generalized Born surface area.

For BACE1 systems, $R^{2}$ by SIE-PM6 and SIE-AM1 are $0.83 \pm 0.02$ and $0.81 \pm 0.02$, respectively, which are better than that by others (Figure 4B). The performance of SIE-PM7 $\left(R^{2}=0.76 \pm 0.01\right)$ is also satisfactory compared with that of SIE-ff03 $\left(R^{2}=0.63 \pm 0.02\right)$. SIE-PM3 produces the worst performance with $R^{2}=0.67 \pm 0.01$ among different SQMPCs, even though it is slightly improved compared with SIE-ff03. Similarly, MM/GBSA-SQMPCs also show better performance than MM/GBSA-ff03 $\left(R^{2}=0.37 \pm 0.01\right)$.

For ligands binding to PDE4D protein, SIE-AM1, SIE-PM6 and SIE-PM7 obtain better results $\left(R^{2}=0.66 \pm 0.02,0.74 \pm 0.02\right.$ and $0.64 \pm 0.02$, respectively) than SIE-ff03 $\left(R^{2}=0.61 \pm 0.02\right)$ (Figure 4C). Similarly, MM/GBSA-PM6 and MM/GBSA-PM7 have better result $\left(R^{2}=0.58 \pm 0.05\right.$ and $0.66 \pm 0.08$, respectively $)$ than MM/GBSA-ff03 $\left(R^{2}=0.53 \pm 0.03\right)$.

For VEGFR2 systems, compared with SIE-ff03 $\left(R^{2}=0.58 \pm 0.01\right)$, SIE-AM1, SIE-PM3, SIE-PM6 and SIEPM7 all achieve satisfactory performance with $R^{2}=0.69 \pm 0.01,0.65 \pm 0.01,0.64 \pm 0.01$ and $0.65 \pm 0.01$, respectively (Figure 4D). Similar results are obtained by using MM/GBSA approach, $R^{2}$ of MM/GBSA-AM1, MM/GBSA-PM3, MM/GBSA-PM6 and MM/GBSA-PM7 $\left(R^{2}=0.65 \pm 0.03,0.63 \pm 0.03,0.52 \pm 0.03\right.$ and $0.51 \pm 0.03$, respectively) are better than MM/GBSA-ff03 $\left(R^{2}=0.41 \pm 0.03\right)$.

Therefore, the study on 50 protein-ligand complexes suggested that $\Delta G$ calculated with MM/GBSA-SQMPC or SIE-SQMPC approach could achieve quite satisfactory performance on diverse protein systems (refer to Supplementary Figure 6 for workflow of MM/GBSA-SQMPC or SIE-SQMPC approach). Among different SQMPC methods, $\mathrm{SQMPC}_{\mathrm{PM} 6}, \mathrm{SQMPC}_{\mathrm{PM} 7}$ and $\mathrm{SQMPC}_{\mathrm{AM} 1}$ show reliable and robust performance, whereas $\mathrm{SQMPC}_{\mathrm{PM} 3}$ 
(A)

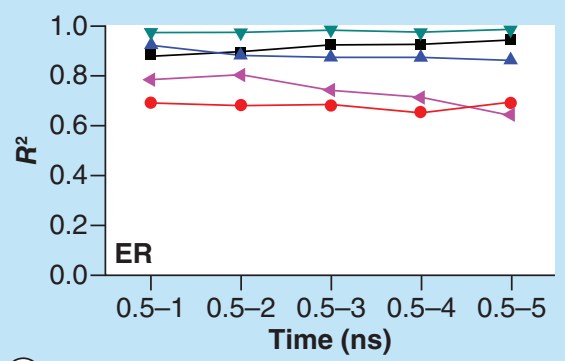

(c)

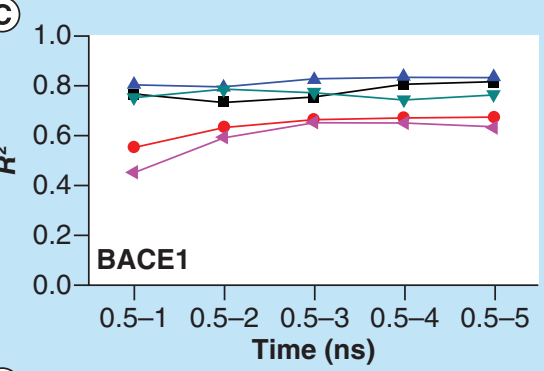

(E)

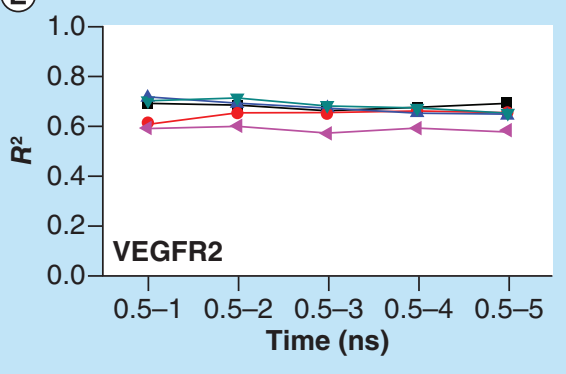

(B)

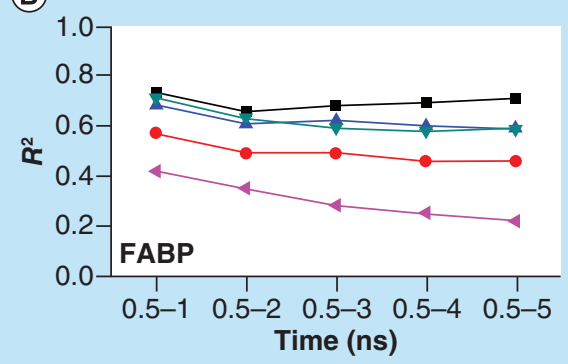

(D)

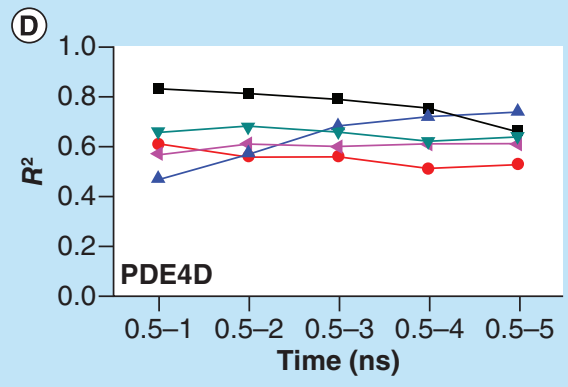

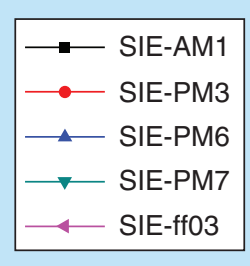

Figure 5. The correlation coefficients calculated based on different molecular dynamics simulation time for different systems. (A) ER, (B) FABP, (C) BACE1, (D) PDE4D and (E) VEGFR2 with SIE-AM1, SIE-PM3, SIE-PM6, SIE-PM7 and SIE-ffO3, respectively.

SIE: Solvated interaction energy.

sometimes produces unsatisfactory predictions, indicating that PM6, PM7 and AM1 might be good choices for SQMPC. Moreover, the performance of SIE is better than that of MM/GBSA on $\triangle G$ calculation with SQMPC in overall. Taking into account that more computational resources required for MM/GBSA and QMPC, SIE-SQMPC should be a better choice for predicting $\Delta G$ of protein-ligand interaction.

It is worth noting that scaffolds of ligands for ER, FABP, PDE4D and VEGFR2 systems are highly diverse as Tanimoto similarity coefficients between them are rather low (Supplementary Figure 7). For example, the Tanimoto similarity coefficients of FABP ligands in complexes 1HMS, 1HMT and 1HMR are around 0.1-0.3, indicating they have very different molecular structures. Thus, these results suggested that SQMPC has strong expansibility on diverse target proteins and ligands with different molecular scaffolds.

It is also observed that the largest improvement of $R^{2}$ achieved by SQMPC is on FABP systems. For exploring its reasons, detailed analysis of ligand structure was performed, revealing that almost all the ligands binding to FABP are deprotonated to form anions (Supplementary Table 1), thus electrostatic polarizations in these systems should be stronger than that in other systems. Therefore, the significant improvement on $R^{2}$ should be attributed to SQMPC, which could properly deal with the electrostatic polarization.

Furthermore, to evaluate how different SQMPCs affect the stability of hydrogen bond, we analyzed the average length of hydrogen bond along MD simulation for all protein-ligand systems (Supplementary Table 6). Compared with the mean length of hydrogen bonds in $\mathrm{x}$-ray crystal structure $(3.02 \AA)$, the hydrogen bonds simulated with $\mathrm{SQMPC}_{\mathrm{AM} 1}, \mathrm{SQMPC}_{\mathrm{PM} 6}$ and $\mathrm{SQMPC}_{\mathrm{PM} 7}(3.50,3.48$ and $3.31 \AA$, respectively) are more stable than 
that with $\mathrm{SQMPC}_{\mathrm{PM} 3}(3.98 \AA$ ) and Amber ff03 charge (4.07 $\AA$ ). A very similar result was obtained from our previous QM/MM MD simulation with PM3, which underestimated hydrogen bond formed between ligand and trypsin [44], suggesting that PM3 might be improper for treating hydrogen bond during MD simulation.

\section{The length of MD simulation impacts the binding-free energy prediction}

To study the influence of MD simulation time on $\Delta G$ calculation by SIE-SQMPC, we carried out a comparative analysis for the predicted $\Delta G$ of the 50 studied protein-ligand systems, using five different lengths of MD simulation, including $0.5-1 \mathrm{~ns}, 0.5-2 \mathrm{~ns}, 0.5-3 \mathrm{~ns}, 0.5-4 \mathrm{~ns}$ and $0.5-5 \mathrm{~ns}$, respectively. As shown in Figure 5, $R^{2}$ is sensitive to the time length of MD simulation. Taking SIE-PM6 as an example, the $R^{2}$ of FABP based on $0.5-1 \mathrm{~ns}$ is the best $\left(R^{2}=0.68 \pm 0.02\right)$ (Figure $5 \mathrm{~B}$ ), while that of PDE4D based on $0.5-1 \mathrm{~ns}$ is the worst $\left(R^{2}=0.47 \pm 0.02\right)$ (Figure $5 \mathrm{D})$. For FABP system, $\Delta G$ prediction based on relatively shorter MD simulation (0.5-1 ns) trajectory is slightly better than that based on longer MD simulation (0.5-5 ns) trajectory. However, for BACE1 system, $\Delta G$ prediction is better with longer MD simulation (Figure 5C). Inconsistent simulation time for the best $R^{2}$ revealed that extending the MD simulation time does not always improve the accuracy for $\Delta G$ prediction, which is consistent with the result reported by Hou et al. [12,68]. Nevertheless, the length of MD simulation time has an obvious impact on $\Delta G$ prediction.

There is no universal length of MD simulation time suitable for all studied protein-ligand systems. We thus analyzed the $R^{2}$ of different SIE-SQMPC approaches using diverse time length of MD simulation. As shown in Figure 6, SIE-AM1 and SIE-PM6 prefer shorter MD simulation time, such as 0.5-1 ns, resulting in high $R^{2}$ in all five target protein systems. By contrast, the prediction with SIE-PM7 is insensitive to the length of MD simulation time, especially for PDE4D system.

\section{Conclusion}

Over the past years, many methods have been proposed to calculate the atomic charges of protein-ligand system for accurate $\Delta G$ prediction. However, a fast but reliable calculation remains a challenge. Fast methods, such as a variety of force fields are questioned in the reliability and expansibility. Although the free energy perturbation and thermodynamic integration are accurate for predicting $\Delta G$ of a series of compounds, both require huge computational resources, thus hampering their application, especially, in high-throughput screening for drug discovery and design [69]. Accordingly, other approaches, for example, LIE, MM/GBSA, MM/PBSA and SIE, have been developed. However, it is still a challenge to achieve a balance between speed and accuracy. We previously developed a method (QMPC) based on the atomic charge of the whole protein-ligand system calculated with QM/MM that could provide a good correlation between the experimentally determined and predicted $\Delta G$ s. Such a method, however, still requires huge computational resources owing to QM calculation at B3LYP/6-31G* level. In this study, we evaluated the performance of atomic charges calculated by using four different semiempirical quantum mechanics methods (AM1, PM3, PM6 and PM7) for predicting $\Delta G$ of 50 protein-ligand systems with SIE and MM/GBSA approaches. First, our results showed that either QMPC or SQMPC achieved better $R^{2}$ than conventional approach (Amber ff03 charge) for all those studied systems. Among them, SQMPC $\mathrm{AM}_{1}$, $\mathrm{SQMPC}_{\mathrm{PM} 6}$ and $\mathrm{SQMPC}_{\mathrm{PM} 7}$ methods could achieve satisfactory performance on diverse systems and consume much less computational resources than QMPC method. Second, the calculated partial atomic charge difference and individual components of $\Delta G$ showed that not only QMPC but also SQMPC method can deal properly with electrostatic polarization more accurate than conventional Amber ff03 method. Third, MD simulation with the atomic charge calculated by the $\mathrm{SQMPC}_{\mathrm{AM} 1}$, $\mathrm{SQMPC}_{\mathrm{PM} 6}$ and $\mathrm{SQMPC}_{\mathrm{PM}}$ methods, but not the $\mathrm{SQMPC}_{\mathrm{PM} 3}$ method, can reproduce important hydrogen bond interaction, which makes a substantial contribution to binding affinity. Fourth, the correlation between the calculated and experimental $\Delta G$ with SQMPC was greatly improved even for ligands with highly diverse scaffolds. Finally, although SIE and MM/GBSA approaches with atomic charge of protein-ligand complex calculated by SQMPC produced similar $R^{2}$ between the predicted and experimentally determined $\Delta G$, SIE is more efficient in terms of consuming computational resources.

\section{Future perspective}

It is a great challenge to accurately predict the $\Delta G$ of ligand to protein. Many researches have been devoted to calculating protein-ligand $\Delta G$ accurately, while obstructed by many factors, one of which is the electrostatic polarization effect. The method proposed in this study for calculating atomic charge of both protein and ligand at semiempirical quantum chemistry level is more likely able to deal well with the electrostatic polarization effect, 
(A)

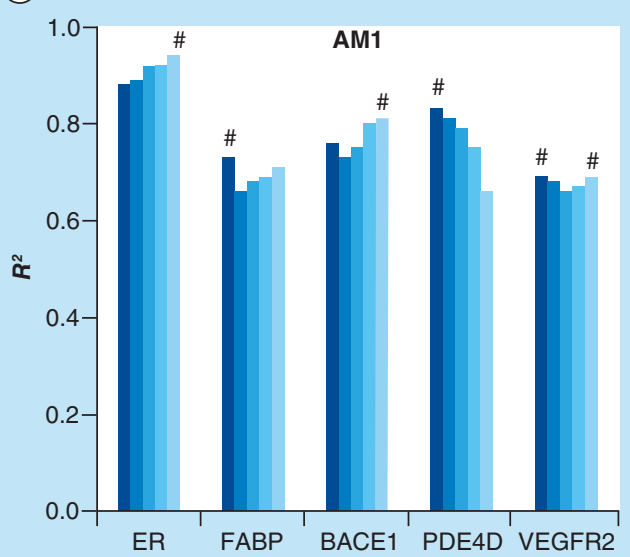

(B)

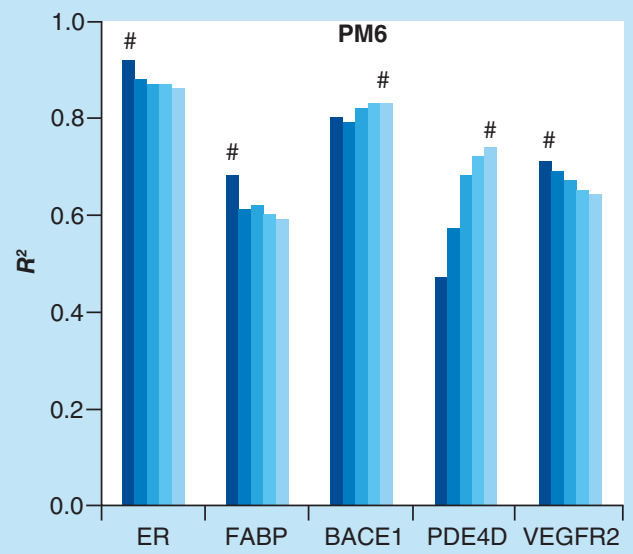

(C)
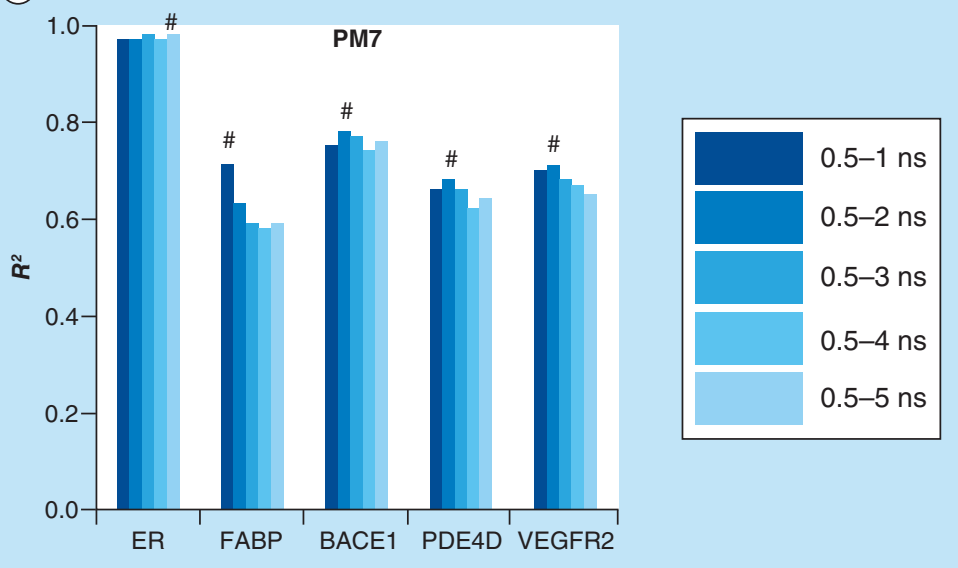

Figure 6. The correlation coefficients with different lengths of molecular dynamics simulation time for the five studied protein systems. (A) SIE-AM1, (B) SIE-PM6 and (C) SIE-PM7.

' $\#$ ' represents the best correlation coefficient.

SIE: Solvated interaction energy.

and improve the correlation between the experimentally determined and predicted $\Delta G$ s with SIE approach. This new approach is also reliable for those ligands with highly diverse scaffolds, which has potential application on high-throughput processing for molecular docking and drug design.

\section{Supplementary data}

See online at: www.future-science.com/doi/full/10.4155/fmc-2018-0207

\section{Financial \& competing interests disclosure}

This work was supported by the National Key R\&D Programs of China (2016YFA0502301 and 2017YFB0202601), and the National Natural Science Foundation of China (81573350). The simulations were partially run at TianHe 1 supercomputer in Tianjing and TianHe 2 supercomputer in Guangzhou, supported by Special Program for Applied Research on Super Computation of the NSFCGuangdong Joint Fund (the second phase) with Grant No.U1501501. The authors have no other relevant affiliations or financial involvement with any organization or entity with a financial interest in or financial conflict with the subject matter or materials discussed in the manuscript apart from those disclosed.

No writing assistance was utilized in the production of this manuscript. 
Ethical conduct of research

The authors state that they have obtained appropriate institutional review board approval or have followed the principles outlined in the Declaration of Helsinki for all human or animal experimental investigations. In addition, for investigations involving human subjects, informed consent has been obtained from the participants involved.

\section{Open access}

This work is licensed under the Attribution-NonCommercial-NoDerivatives 4.0 Unported License. To view a copy of this license, visit http://creativecommons.org/licenses/by-nc-nd/4.0/

\section{Summary points}

\section{Background}

- The accurate prediction of protein-ligand binding-free energy is of significance for drug design and development, which is, however, very difficult, especially for a series of ligands with highly diverse scaffolds.

- The accuracy of binding-free energy calculated with both molecular mechanics/Poisson-Boltzmann (or generalized Born) surface area and solvated interaction energy (SIE) approaches is greatly influenced by the treatment of atomic charges of both ligand and protein.

- The QMPC approach developed previously could calculate atomic charge of protein precisely with quantum mechanics/molecular mechanics and deal well with electrostatic polarization effect, which is however limited by its expensive computational resources for quantum mechanics/molecular mechanics calculation.

- The accuracy of semiempirical quantum mechanics for noncovalent interactions is comparable to dispersion-corrected density functional theory, while its computational time is much less.

Method

- A new approach, named SQMPC, was developed in this study to calculate atomic charge of both protein and ligand, which could combine with SIE method, namely SIE-SQMPC, to calculate protein-ligand binding-free energy.

\section{Conclusion}

- The results showed that SIE-SQMPC achieved much better correlation coefficient than SIE-ff03 charge for all studied systems. Among them, SIE-AM1 and SIE-PM7 could always achieve a good correlation between the predicted and experimental binding-free energy with short molecular dynamics simulation time ( $\leq 2 \mathrm{~ns})$.

- The results showed that not only QMPC but also SQMPC method can deal properly with the electrostatic contribution on ligand-binding comparing with conventional Amber ff03 method.

- Unexpectedly, longer molecular dynamics simulation is not always necessary to accomplish better prediction. It should be an open question for further discussion.

- SIE-SQMPC approach provides an alternative to accurately predict binding-free energy.

\section{References}

Papers of special note have been highlighted as: $\bullet$ of interest; $\bullet \bullet$ of considerable interest

1. Genheden S, Ryde U. The MM/PBSA and MM/GBSA methods to estimate ligand-binding affinities. Expert Opin. Drug Discov. 10(5), 449-461 (2015).

2. Ryde U, Söderhjelm P. Ligand-binding affinity estimates supported by quantum-mechanical methods. Chem. Rev. 116(9), 5520-5566 (2016).

3. Steinbrecher TB, Dahlgren M, Cappel D et al. Accurate binding free energy predictions in fragment optimization. J. Chem. Inf. Model. 55(11), 2411-2420 (2015).

4. Ferreira LG, Dos Santos RN, Oliva G, Andricopulo AD. Molecular docking and structure-based drug design strategies. Molecules 20(7), 13384-13421 (2015).

5. Jorgensen WL. Free energy calculations: a breakthrough for modeling organic chemistry in solution. Acc. Chem. Res. 22(5), 184-189 (1989).

6. Wong CF, McCammon JA. Dynamics and design of enzymes and inhibitors. J. Am. Chem. Soc. 108(13), 3830-3832 (1986).

7. Hansson T, Marelius J, Åqvist J. Ligand binding affinity prediction by linear interaction energy methods. J. Comput. Aided Mol. Des. 12(1), 27-35 (1998).

8. Åqvist J, Medina C, Samuelsson JE. A new method for predicting binding affinity in computer-aided drug design. Protein Eng. 7(3), 385-391 (1994).

9. Kollman PA, Massova I, Reyes C et al. Calculating structures and free energies of complex molecules: combining molecular mechanics and continuum models. Acc. Chem. Res. 33(12), 889-897 (2000). 
10. Srinivasan J, Cheatham TE, Cieplak P, Kollman PA, Case DA. Continuum solvent studies of the stability of DNA, RNA, and phosphoramidate-DNA helices. J. Am. Chem. Soc. 120(37), 9401-9409 (1998).

11. Homeyer N, Gohlke H. Free energy calculations by the molecular mechanics Poisson-Boltzmann surface area method. Mol. Inf. 31(2), 114-122 (2012).

12. Xu L, Sun H, Li Y, Wang J, Hou T. Assessing the performance of MM/PBSA and MM/GBSA methods. 3. The impact of force fields and ligand charge models. J. Phys. Chem. B 117(28), 8408-8421 (2013).

- Paper describes the factors impacting the performance of molecular mechanics/Poisson-Boltzmann surface area and molecular mechanics/generalized Born surface area methods.

13. Davis ME, McCammon JA. Electrostatics in biomolecular structure and dynamics. Chem. Rev. 90(3), 509-521 (1990).

14. Weis A, Katebzadeh K, Söderhjelm P, Nilsson I, Ryde U. Ligand affinities predicted with the MM/PBSA method: dependence on the simulation method and the force field. J. Med. Chem. 49(22), 6596-6606 (2006).

15. Naim M, Bhat $\mathrm{S}$, Rankin $\mathrm{KN}$ et al. Solvated interaction energy (SIE) for scoring protein-ligand binding affinities. 1. Exploring the parameter space. J. Chem. Inf. Model. 47(1), 122-133 (2007).

-• Paper describes the solvated interaction energy approach, which was the main approach to calculate binding-free energy in this study.

16. Salla IV, Sanna PN, Olli TP. Case-specific performance of MM-PBSA, MM-GBSA, and SIE in virtual screening. J. Mol. Graphics Model. 62, 303-318 (2015).

17. Cui Q, Sulea T, Schrag JD et al. Molecular dynamics - solvated interaction energy studies of protein-protein interactions: the MP1-p14 scaffolding complex. J. Mol. Biol. 379(4), 787-802 (2008).

18. Sulea T, Cui Q, Purisima EO. Solvated interaction energy (SIE) for scoring protein-ligand binding affinities. 2. Benchmark in the CSAR-2010 scoring exercise. J. Chem. Inf. Model. 51(9), 2066-2081 (2011).

19. Hogues H, Sulea T, Purisima EO. Evaluation of the Wilma-SIE virtual screening method in community structure-activity resource 2013 and 2014 blind challenges. J. Chem. Inf. Model. 56(6), 955-964 (2015).

20. Sulea T, Hogues H, Purisima EO. Exhaustive search and solvated interaction energy (SIE) for virtual screening and affinity prediction. J. Comput. Aided Mol. Des. 26(5), 617-633 (2012).

21. Hogues H, Sulea T, Purisima EO. Exhaustive docking and solvated interaction energy scoring: lessons learned from the SAMPL4 challenge. J. Comput. Aided Mol. Des. 28(4), 417-427 (2014).

22. Ji C, Mei Y. Some practical approaches to treating electrostatic polarization of proteins. Acc. Chem. Res. 47(9), 2795-2803 (2014).

- Interesting paper in the field of electrostatic polarization effect.

23. Ji C, Mei Y, Zhang JZ. Developing polarized protein-specific charges for protein dynamics: MD free energy calculation of pKa shifts for Asp 26/Asp 20 in Thioredoxin. Biophys. J. 95(3), 1080-1088 (2008).

24. Tong Y, Mei Y, Li YL, Ji CG, Zhang JZ. Electrostatic polarization makes a substantial contribution to the free energy of avidin-biotin binding. J. Am. Chem. Soc. 132(14), 5137-5142 (2010).

25. Mei Y, Li YL, Zeng J, Zhang JZ. Electrostatic polarization is critical for the strong binding in streptavidin-biotin system. J. Comput. Chem. 33(15), 1374-1382 (2012).

26. Cho AE, Guallar V, Berne BJ et al. Importance of accurate charges in molecular docking: quantum mechanical/molecular mechanical (QM/MM) approach. J. Comput. Chem. 26(9), 915-931 (2005).

27. Ponder JW, Wu C, Ren P et al. Current status of the AMOEBA polarizable force field. J. Phys. Chem. B 114(8), 2549-2564 (2010).

28. Piquemal J-P, Jordan KD. From quantum mechanics to force fields: new methodologies for the classical simulation of complex systems. Theor. Chem. Acc. 131, 1-2 (2012).

29. Donchev A, Ozrin V, Subbotin M, Tarasov O, Tarasov V. A quantum mechanical polarizable force field for biomolecular interactions. Proc. Natl Acad. Sci. USA 102(22), 7829-7834 (2005).

30. Khoruzhii O, Donchev AG, Galkin $\mathrm{N}$ et al. Application of a polarizable force field to calculations of relative protein-ligand binding affinities. Proc. Natl Acad. Sci. USA 105(30), 10378-10383 (2008).

31. Jiao D, Golubkov PA, Darden TA, Ren P. Calculation of protein-ligand binding free energy by using a polarizable potential. Proc. Natl Acad. Sci. USA 105(17), 6290-6295 (2008).

32. Cieplak P, Dupradeau F-Y, Duan Y, Wang J. Polarization effects in molecular mechanical force fields. J. Phys. 21(33), 333102 (2009).

33. Söderhjelm P. Polarization effects in protein-ligand calculations extend farther than the actual induction energy. Theo. Chem. Acc. 131(3), 1159 (2012).

34. Chen X, Zhang D, Zhang J. Fractionation of peptide with disulfide bond for quantum mechanical calculation of interaction energy with molecules. The J. Chem. Phys. 120(2), 839-844 (2004).

35. Zhang DW, Chen X, Zhang JZ. Molecular caps for full quantum mechanical computation of peptide-water interaction energy. J. Chem. Phys. 24(15), 1846-1852 (2003). 
36. Polkosnik W, Massa L. Single determinant N-representability and the kernel energy method applied to water clusters. J. Comput. Chem. 39(17), 1038-1043 (2018).

37. Huang L, Matta CF, Massa L. The kernel energy method (KEM) delivers fast and accurate QTAIM electrostatic charge for atoms in large molecules. Struct. Chem. 26(5-6), 1433-1442 (2015).

38. Huang L, Massa L, Krupkin M, Bashan A, Yonath A. Protoribosome by quantum kernel energy method. Proc. Natl Acad. Sci. USA 110(37), 14900-14905 (2013).

39. Matta CF, Massa L. Subsystem quantum mechanics and in silico medicinal and biological chemistry. Future Med. Chem. 3(16), 1971-1974 (2011).

40. Huang L, Massa L, Karle J. Kernel energy method illustrated with peptides. Int. J. Quantum Chem. 103(6), 808-817 (2005).

41. Wang J, Shao Q, Cossins BP, Shi J, Chen K, Zhu W. Thermodynamics calculation of protein-ligand interactions by QM/MM polarizable charge parameters. J. Biomol. Struct. Dyn. 34(1), 163-176 (2016).

-• Of considerable interest since it proposes the QMPC method.

42. Yilmazer ND, Korth M. Enhanced semiempirical QM methods for biomolecular interactions. Comput. Struct. Biotechnol. J. 13, 169-175 (2015).

43. Dral PO, Wu X, Spörkel L, Koslowski A, Thiel W. Semiempirical quantum-chemical orthogonalization-corrected methods: benchmarks for ground-state properties. J. Chem. Theor. Comput. 12(3), 1097-1120 (2016).

44. Chen J, Wang J, Zhang Q, Chen K, Zhu W. A comparative study of trypsin specificity based on QM/MM molecular dynamics simulation and QM/MM GBSA calculation. J. Biomol. Struct. Dyn. 33(12), 2606-2618 (2015).

45. Bikadi Z, Hazai E. Application of the PM6 semi-empirical method to modeling proteins enhances docking accuracy of AutoDock. J. Cheminform. 1(1), 15 (2009).

46. Dewar MJ, Zoebisch EG, Healy EF, Stewart JJ. Development and use of quantum mechanical molecular models. 76. AM1: a new general purpose quantum mechanical molecular model. J. Am. Chem. Soc. 107(13), 3902-3909 (1985).

47. Stewart JJ. Optimization of parameters for semiempirical methods I. Method. J. Comput. Chem. 10(2), $209-220$ (1989).

48. Stewart JJ. Optimization of parameters for semiempirical methods II. Applications. J. Comput. Chem. 10(2), 221-264 (1989).

49. Stewart JJ. Optimization of parameters for semiempirical methods V: modification of NDDO approximations and application to 70 elements. J. Mol. Model. 13(12), 1173-1213 (2007).

50. Stewart JJ. Optimization of parameters for semiempirical methods VI: more modifications to the NDDO approximations and re-optimization of parameters. J. Mol. Model. 19(1), 1-32 (2013).

51. Anandakrishnan R, Aguilar B, Onufriev AV. H++ 3.0: automating $\mathrm{pK}$ prediction and the preparation of biomolecular structures for atomistic molecular modeling and simulations. Nucleic Acids Res. 40(W1), W537-W541 (2012).

52. Wang J, Wolf RM, Caldwell JW, Kollman PA, Case DA. Development and testing of a general amber force field. J. Comput. Chem. 25(9), 1157-1174 (2004).

53. Duan $\mathrm{Y}, \mathrm{Wu} \mathrm{C}$, Chowdhury $\mathrm{S}$ et al. A point-charge force field for molecular mechanics simulations of proteins based on condensed-phase quantum mechanical calculations. J. Comput. Chem. 24(16), 1999-2012 (2003).

54. Wang J, Wang W, Kollman PA, Case DA. Automatic atom type and bond type perception in molecular mechanical calculations. J. Mol. Graphics Model. 25(2), 247-260 (2006).

55. Frisch M, Scuseria G, Robb M et al. Gaussian 09 (Revision C.01) Gaussian Inc, Wallingford (2010).

56. Rocchia W, Sridharan S, Nicholls A, Alexov E, Chiabrera A, Honig B. Rapid grid-based construction of the molecular surface and the use of induced surface charge to calculate reaction field energies: applications to the molecular systems and geometric objects. J. Comput. Chem. 23(1), 128-137 (2002).

57. Cieplak P, Cornell WD, Bayly C, Kollman PA. Application of the multimolecule and multiconformational RESP methodology to biopolymers: charge derivation for DNA, RNA, and proteins. J. Comput. Chem. 16(11), 1357-1377 (1995).

58. Bayly CI, Cieplak P, Cornell W, Kollman PA. A well-behaved electrostatic potential based method using charge restraints for deriving atomic charges: the RESP model. J. Phys. Chem. 97(40), 10269-10280 (1993).

59. Maia JDC, Urquiza Carvalho GA, Mangueira CP Jr, Santana SR, Cabral La, Rocha GB. GPU linear algebra libraries and GPGPU programming for accelerating MOPAC semiempirical quantum chemistry calculations. J. Chem. Theor. Comput. 8(9), 3072-3081 (2012).

60. Stewart JJP. MOPAC2016 (2016).

61. Ryckaert J-P, Ciccotti G, Berendsen HJ. Numerical integration of the cartesian equations of motion of a system with constraints: molecular dynamics of n-alkanes. J. Comput. Phys. 23(3), 327-341 (1977).

62. Essmann U, Perera L, Berkowitz ML, Darden T, Lee H, Pedersen LG. A smooth particle mesh Ewald method. J. Chem. Phys. 103(19), 8577-8593 (1995). 
63. Onufriev A, Bashford D, Case DA. Exploring protein native states and large-scale conformational changes with a modified generalized born model. Proteins 55(2), 383-394 (2004).

64. Sanner MF, Olson AJ, Spehner JC. Reduced surface: an efficient way to compute molecular surfaces. Biopolymers 38(3), 305-320 (1996).

65. Purisima EO, Nilar SH. A simple yet accurate boundary element method for continuum dielectric calculations. J. Comput. Chem. 16(6), 681-689 (1995).

66. Purisima EO. Fast summation boundary element method for calculating solvation free energies of macromolecules. J. Comput. Chem. 19(13), 1494-1504 (1998).

67. Bhat S, Purisima EO. Molecular surface generation using a variable-radius solvent probe. Proteins 62(1), $244-261$ (2006).

68. Hou T, Wang J, Li Y, Wang W. Assessing the performance of the MM/PBSA and MM/GBSA methods. 1. The accuracy of binding free energy calculations based on molecular dynamics simulations. J. Chem. Inf. Model. 51(1), 69-82 (2010).

69. Pu C, Yan G, Shi J. Assessing the performance of docking scoring function, FEP, MM-GBSA, and QM/MM-GBSA approaches on a series of PLK1 inhibitors. Med. Chem. Commun. 8(7), 1452-1458 (2017). 
